\title{
La pénétration des manuels scolaires de France au Québec
}

Un cas-type : Les frères des Écoles chrétiennes, XIXe-XXe siècles

\section{Paul Aubin}

\section{OpenEdition}

\section{Journals}

\section{Édition électronique}

URL : https://journals.openedition.org/histoire-education/1232

DOI : $10.4000 /$ histoire-education. 1232

ISSN : 2102-5452

Éditeur

ENS Éditions

\section{Édition imprimée}

Date de publication : 1 janvier 2000

Pagination : $3-24$

ISBN : 2-7342-0668-4

ISSN : 0221-6280

\section{Référence électronique}

Paul Aubin, «La pénétration des manuels scolaires de France au Québec », Histoire de l'éducation [En ligne], 85 | 2000, mis en ligne le 23 mars 2009, consulté le 20 mai 2021. URL : http://

journals.openedition.org/histoire-education/1232 ; DOI : https://doi.org/10.4000/histoire-education. 1232

Ce document a été généré automatiquement le 20 mai 2021.

(c) Tous droits réservés 


\section{La pénétration des manuels scolaires de France au Québec}

Un cas-type : Les frères des Écoles chrétiennes, XIXe-XXe siècles

\section{Paul Aubin}

1 Dans son aventure missionnaire, l'Église catholique, tout comme les autres, a vu dans l'école un moyen privilégié de répandre sa vision du monde. Non seulement elle a favorisé l'émigration, de l'Europe vers les nouvelles contrées, de structures déjà éprouvées pour favoriser cette expansion, mais elle a aussi exporté des instruments pédagogiques dont l'utilité était depuis longtemps éprouvée pour la transmission de la culture catholique d'expression française. Mais pourquoi un tel transfert lorsque le pays récepteur parlait la même langue et professait la même religion que le pays émetteur? Collectivité majoritairement franco-catholique, le Québec avait-il besoin d'importer des manuels scolaires de France ? Or pendant deux cents ans, on y a utilisé dans l'enseignement pré-universitaire des centaines de livres publiés initialement en France. L'aventure, en terre québécoise, de la communauté des frères des Écoles chrétiennes illustre ce phénomène et fournit un certain nombre d'explications ${ }^{1}$.

\section{L'importation des manuels des frères au Québec}

2 Fondée en France à la fin du XVIIe siècle, la communauté des frères des Écoles chrétiennes adresse son enseignement aux garçons des classes populaires en milieu urbain. Fortement ébranlée par la Révolution française, elle renaît dans le courant du renouveau religieux en France au début du XIXe siècle et se lance dans une politique d'expansion. En France, la communauté avait produit des manuels scolaires dès ses origines : on doit notamment à son fondateur, le chanoine rémois Jean-Baptiste de La Salle, entre autres publications, un Traité des devoirs du chrétien qui connaîtra 150 ans plus tard une grande diffusion au Québec en tant que livre de lecture.

3 À peine implantée à Montréal où arrivent quatre frères français en novembre $1837^{2}$, la communauté fait publier un premier traité d'arithmétique au printemps $1838^{3}$. Utilisant d'abord le réseau local des éditeurs-imprimeurs-libraires, elle s'identifie 
formellement comme maison d'édition en 1869 - la maison-mère de Paris attendra le début du XXe siècle pour se lancer dans l'édition, - se dote d'une imprimerie en 1890 et occupe durant la première moitié du XXe siècle une place de choix dans le monde de l'édition scolaire au Québec. À partir de 1960, sa maison d'édition, désormais identifiée sous le sigle Lidec, acronyme de Librairie des écoles, connait de profonds changements, témoins des bouleversements de la société québécoise, jusqu'à sa liquidation, en 1985, en faveur de l'éditeur Guérin. Si les frères transplantés au Québec en viennent très tôt à rédiger et publier leurs propres manuels, il n'en demeure pas moins qu'ils peuvent compter sur un important fonds original en France - les publications de leurs confrères - et ils ne s'en privent pas. Cette présence au Québec du manuel d'outre-mer se présente sous différentes façons.

\section{Importations en feuilles}

4 On trouve trace, uniquement au XIXe siècle, de l'envoi d'une demi-douzaine de manuels rédigés par des frères français, imprimés en France et expédiés en feuilles pour être assemblés et reliés au Québec. Le frère Aidant, premier directeur à Montréal, écrit au supérieur général à Paris quatre mois après son arrivée: "J'ai fait part à $\mathrm{Mr}$ le Supérieur de St Sulpice, des raisons qui vous empêchaient de nous envoyer la Géographie ; il ne les trouve pas suffisantes pour priver nos jeunes Canadiens d'un aussi précieux livre ; en conséquence, il m'a prié de faire une nouvelle demande en feuilles, on supprimera avant de la faire relier, ce qui regarde la France et il fera imprimer une ou deux feuilles pour le remplacer, où il sera parlé avec développement de l'Angleterre et de ses possessions Américaines, Africaines, etc., ainsi que des États-Unis voisins du Canada. $»^{4}$

5 L'examen des exemplaires des Éléments de trigonométrie (1875) et de l'Arpentage - levé des plans (1875) conservés à la Bibliothèque nationale du Canada étaye cette hypothèse : le papier de la page de titre, sur laquelle apparait le nom de l'imprimeur-libraire québécois Elzéar Vincent, est différent de celui du reste du livre et de qualité nettement inférieure; dans les deux cas, le colophon indique "Tours, impr. Mame». Mais cette pratique semble sur le point de disparaître dans les années 1880 et le supérieur local ne manque pas d'en souligner les inconvénients : «Si les ouvrages français pris en feuilles à Paris nous étaient cédés au même prix que nous pourrions les avoir en les imprimant ici, il nous en faudrait un stock considérable que nous ne saurions où loger ; les avaries, les déterriorations [sic] gâteraient un bon nombre de volumes, ce qui augmenterait le prix des autres ouvrages. $\|^{5}$ Indice à la fois du besoin de combler rapidement des vides, $\mathrm{du}$ manque d'auteurs locaux dans certaines disciplines pointues tout comme de l'exiguïté d'un marché ne justifiant pas l'investissement requis pour la réécriture de tels titres, ce type de présence du manuel français au Québec est occasionnel: il comportait trop de risques quant aux aléas de l'expédition par bateaux ${ }^{6}$.

\section{Réimpressions intégrales}

6 C'est surtout par les réimpressions intégrales d'ouvrages de leurs confrères de France que les frères du Québec font sentir la présence de l'ancienne mère-patrie. Des 119 titres que la communauté publie au XIXe siècle, 26 , soit $21 \%$, sont des réimpressions intégrales de manuels de France; et ce pourcentage grimpe à $38 \%$ si l'on ne tient pas compte de certaines catégories de manuels - histoire du Canada, grammaire anglaise, 
etc. - qui ne pouvaient compter sur une source étrangère. Au XXe siècle, la situation change du tout au tout: des 295 titres que publie la communauté entre 1900 et 1965, 19 seulement sont des réimpressions en terre québécoise de manuels rédigés par leurs confrères de France, et encore la plupart l'ont-ils été durant la Deuxième Guerre mondiale.

Que la majeure partie des réimpressions de manuels de France se retrouve dans le secteur de l'enseignement $d u$ français n'a rien pour surprendre. Ainsi, les douze premières grammaires publiées entre 1842 et 1881 sont toutes des reproductions intégrales de manuels français; un des quatre syllabaires (Syllabaire ou premiers exercices de lecture en rapport avec la méthode d'écriture des frères des écoles chrétiennes, 1875) et les deux premiers livres de lecture (Lectures instructives et amusantes, 1864, et Lectures courantes faisant suite au premier livre de lecture, 1875, auxquels il faut ajouter le Nouveau traité des devoirs du chrétien, 1841, toujours catalogué et utilisé comme livre de lecture) et leur seul dictionnaire (Petit dictionnaire ou lexique orthographique mis en rapport avec la dernière édition du dictionnaire de l'Académie, c. 1895). Du côté des mathématiques, la tendance à reproduire des livres de France est moins marquée : un manuel de calcul (Exercices de calcul sur les quatre opérations fondamentales de l'arithmétique, c. 1873) sur six, les cinq autres se présentant sous forme de cahiers d'exercices rédigés au Québec par un frère français, les deux premiers des onze manuels d'arithmétique (Nouveau traité d'arithmétique contenant toutes les opérations ordinaires de calcul [...], 1838 et Nouvelle arithmétique analytique et synthétique des académies, des écoles modèles et commerciales [...], 1858), les deux manuels de trigonométrie et d'arpentage déjà mentionnés tout en rappelant que, pour ces deux derniers, il s'agit vraisemblablement de manuels imprimés en France mais assemblés et reliés au Québec. La géographie ne fait l'objet que d'une réimpression intégrale de manuel français (Abrégé de géographie commerciale et historique, suivi d'un précis de cosmographie selon le système de Copernic [...], 1842) - il pourrait s'agir du manuel de géographie dont le frère Aidant avait demandé, en vain semble-t-il, l'envoi en feuilles - alors que le seul traité rédigé en français sur l'histoire sainte au XIXe siècle (Cours moyen d'histoire sainte à l'usage des écoles chrétiennes, c. 1884) vient également de France. Il va de soi que les deux œuvres dues à la plume du fondateur - (Les règles de la bienséance et de la civilité chrétienne: publié deux fois au Québec avant l'arrivée des frères, ce texte sera ensuite systématiquement joint au Nouveau traité des devoirs du chrétien déjà cité) de même que la version anglaise de ce dernier (A new treatise of the duties of a christian, 1862) - quoique fortement remaniées, sont comptabilisées avec les publications d'origine française.

8 L'influence de la France dans les manuels scolaires de la branche québécoise diminue sensiblement au XXe siècle. Les chiffres, en nombre absolu, risquent de nous induire en erreur, car on passe de 23 réimpressions intégrales au siècle précédent à 19 , d'où l'impression, à première vue, d'un continuum dans le rôle de la métropole (ce terme conserve son sens en ce qui regarde la communauté); dans les faits, il en va tout autrement: sur les 295 titres que publie la congrégation entre 1900 et 1965, les 19 manuels français réimprimés ou diffusés au Canada ne représentent plus que $0,06 \%$. De plus, 14 de ces 19 titres, publiés une première fois entre 1941 et 1942, le sont en conséquence des conditions du marché liées directement à la guerre et s'adressent à des niveaux scolaires que ne rejoignaient pas la plupart des publications antérieures des frères canadiens ${ }^{7}$; le Cours de géométrie publié en 1964 peut être rattaché au « cycle de guerre » car il s'agit, là encore, d'un manuel rédigé par leurs confrères de France, 
sauf que dans ce cas nous avons affaire à une coédition entre les maisons d'édition des frères de France et celle du Québec et dont l'impression est faite en France. Cet ensemble avait été précédé, en 1919 , du Dictionnaire du bon langage ${ }^{8}$ du prêtre québécois Étienne Blanchard et du Petit dictionnaire français en 1922. N'a pas été retrouvé le manuel français dont les Exercices de calcul sur les quatre opérations fondamentales de l'arithmétique serait une copie conforme ${ }^{9}$. Finalement, la procure de Montréal, sous la raison sociale Lidec, s'associe à Mame et Hatier, en 1967, pour publier un Dictionnaire usuel du français moderne - augmenté d'une section historique et géographique propre au Canada dont un des trois auteurs est le frère québécois Raymond Brisebois.

\section{Adaptations}

9 Entre les réimpressions intégrales et les productions du cru, il y avait place pour des adaptations de textes rédigés en Europe auxquels on faisait subir les nécessaires transformations pour les rendre intelligibles ici, comme ce manuel d'arithmétique de 1838 déjà cité dans lequel le système métrique cède la place aux mesures à l'anglaise ${ }^{10}$. Mais c'est le Syllabaire - premier livre de 1872 (144 p.) qui illustre le mieux le processus d'adaptation. Des 23 premières leçons qui le composent, 22 sont très proches d'un modèle français beaucoup plus court, publié antérieurement en France et que la communauté fait paraître in extenso à Québec en 1875 : Syllabaire ou premiers exercices de lecture en rapport avec la méthode d'écriture des frères des écoles chrétiennes (36 p. $)^{11}$. Dans bien des cas, les différences se résument à des formulations légèrement modifiées: «Études des sons de deux lettres » de l'original français (p. 20) plutôt que «Études des sons simples représentés par deux lettres » (p.18) dans la version québécoise; dans d'autres cas, on modifie légèrement le texte du syllabaire français pour teinter le syllabaire québécois de références locales: ainsi, dans la leçon 58 sur les «Objets alimentaires » on précise que "Nous avons, au Canada, tout ce qu'on peut désirer sous le rapport de la nourriture : un excellent pain et de la viande en abondance ". La réédition de 1891 du même syllabaire québécois de 1872 voit apparaître de nouvelles modifications en regard du modèle français de 1875 : «le cler-gé cha-ri-ta-ble » de la version française (p. 17) devient « le cler-gé cha-ri-ta-ble de ces lieux » en 1891 (p. 27); ailleurs, il suffit de simples ajouts pour donner l'impression que le texte s'adresse bien à la population locale: "L'or-gue de la ba-si-li-que » de la version française originale (p.15) est remplacé par "L'or-gue de la ba-si-li-que de Qué-bec» en 1891; une évolution similaire se dessine dans le choix des illustrations: la gravure de la Sainte famille au début du Syllabaire français de 1875 cède la place à celle de Jacques Cartier dans le Syllabaire de 1891 ; on note également un effort pour démarquer le Syllabaire de 1891 de son modèle français par l'introduction de certains textes de la littérature québécoise, comme en font foi des extraits de "La légende d'un peuple » de Louis Fréchette ${ }^{12}$ et de «Le Canada au XIXe siècle » d'A.-B. Routhier. Finalement, le seul syllabaire publié par les frères de Montréal au XXe siècle, Lecture par la méthode phonique - première partie de 1918, réédité en 1935 sous le titre Cours de lecture - premier livre (par la méthode phonique) révèle une nette volonté d'identification au contexte local, ne serait-ce que par une illustration d'un joueur de hockey ou la mention de la capitale fédérale; il n'en demeure pas moins que ce manuel puise dans les syllabaires français antérieurs, de l'aveu même du supérieur de Montréal ${ }^{13}$.

10 Signalons, à titre d'exemples, d'autres cas d'adaptation. Ainsi, en grammaire. A partir de 1882, les frères du Québec cessent de réimprimer des livres de France de cette 
discipline, pour rédiger les leurs, sans pour autant s'affranchir totalement comme en témoigne la définition de la grammaire empruntée au manuel français de 1881, Leçons de lexicologie - cours élémentaire et qui se retrouve mot à mot dans la majorité des grammaires québécoises et ce jusqu'en 1950; mais il faudrait plutôt parler de méthodologie empruntée aux manuels français que de longs extraits textuels. Autre exemple d'adaptation, cette fois en géographie, où l'exemple accolé à la définition du terme « île » passe de l'île Saint-Louis dans la version française à l'île d'Orléans, près de Québec (Géographie du cours élémentaire ou inférieur à l'usage des écoles chrétiennes, 1873). Si certains élèves doutaient encore de la source métropolitaine, jusqu'à cette date, des géographies des frères, ils n'avaient qu'à lire la préface du Manuel de géographie élémentaire de 1874 : «En publiant Manuel de géographie, notre intention n'est point de présenter un ouvrage nouveau, mais plutôt une nouvelle édition de notre Géographie imprimée dès le commencement de nos établissements au Canada [...] modelé en grande partie sur celui du frère Alexis, professeur de Géographie à l'École Normale de Belgique, et auteur de plusieurs ouvrages très répandus, tant en Belgique qu'en France ».

11 Ce mode d'appropriation - pour ne pas dire maquillage dans certains cas - est surtout utilisé au XIXe siècle, les auteurs québécois prenant de plus en plus leurs distances avec le modèle européen par la suite.

12 La dernière adaptation pour le marché québécois de manuels français, et non la moindre, est l'Histoire sainte - cours moyen qui fait carrière de 1921 à 1959 : adaptation on prend la peine d'indiquer que le manuel de 1921 est une "Nouvelle édition illustrée » - d'un titre presque identique publié pour la première fois au Québec en 1884 et dont l'auteur était français (la page de titre porte les initiales F.I.C., soit frère Irlide Cazaneuve, supérieur général de la communauté à Paris).

\section{Importations de livres reliés}

13 Dans l'étude des transferts de littérature didactique, il faut tenir compte de l'importation de livres publiés en France et utilisés au Québec sans y être réimprimés. L'enseignement du dessin industriel illustre ce propos. Dès 1878, le supérieur montréalais tente, sans succès, de faire approuver la méthode de dessin utilisée par sa communauté en France ${ }^{14}$. Or le défaut d'approbation d'un manuel n'en interdit pas, il s'en faut, son utilisation. Ainsi, lorsqu'en 1891 on procède auprès des inspecteurs d'école à une enquête sur l'enseignement de cette discipline, cinq des trente-trois réponses reçues indiquent clairement l'utilisation du manuel de la communauté pour l'enseignement du dessin, l'inspecteur de Louiseville précisant qu'on y fait usage de " cahiers imprimés par les Frères des Écoles chrétiennes " ${ }^{15}$. Comme on ne trouve trace nulle part de la moindre impression de manuels de cette discipline par les frères au Québec, il nous faut conclure qu'ils utilisaient des ouvrages importés ${ }^{16}$.

Lorsque leurs confrères de France fondent leur propre maison d'édition - Ligel, acronyme de Librairie générale de l'enseignement libre -, l'exportation ne fait que s'amplifier. Il semble que cette exportation de la production des frères des Écoles chrétiennes de France ait trouvé au Québec une clientèle intéressante à partir de 1950; au frère Clément, directeur de la procure de Montréal qui lui offre de devenir le seul intermédiaire au Canada pour la communauté, le frère Charles, directeur de Ligel, répond en 1958 : « Nous avons noté quant à nous, en effet, un accroissement important 
de nos relations commerciales avec le Canada et nous en sommes fort contents. [...] Nous avons un intérêt majeur à faire de l'exportation. Nous sommes inscrits parmi les meilleurs exportateurs de manuels français par les organismes officiels de notre pays. Si nous renoncions à nos exportations au Canada, nous risquerions de perdre le rang que nous détenons et que nous souhaitons améliorer encore ; il en résulterait pour nous de graves préjudices. Nous aurions en outre des conflits incessants avec nos confrères éditeurs. Les maisons Hachette, de Ginkgo, L'Écolier nous font en effet des commandes importantes pour le Canada, il ne nous est pas possible de leur dire que nous avons donné une exclusivité à l'un quelconque des libraires de votre pays. Si, par ailleurs, on considère le montant de nos ventes à la seule ville de Montréal, on constate que les factures établies à votre Procure de Montréal ne représentent pas le tiers de celles des libraires ou autres organismes de votre ville. Comment voulez-vous que, dans ces conditions, nous déclarions à ces gros clients que désormais nous ne pourrons plus les servir qu'ils devront s'adresser à vous? Nous risquerions de les rebuter et de les engager ainsi à chercher et conseiller d'autres manuels que les nôtres. Nous ne servirions ainsi ni vos intérêts ni les nôtres. Ne croyez-vous pas que la formule ne réside pas dans une édition faite par vous de nos livres, ni dans une exclusivité accordée à votre Procure, mais plutôt dans une prospection plus active de votre part des clients qu'intéressent nos ouvrages pour que ces clients s'adressent à vous au lieu de commander nos manuels à tel ou tel libraire de Montréal ou d'ailleurs? Nous pensons - et l'expérience identique réalisée dans un autre pays très éloigné du vôtre nous montre que notre politique est bonne - nous pensons que vous pouvez accroître considérablement votre chiffre d'affaires en vendant nos livres, sans que vous les ayez vous-mêmes édités, les conditions que nous faisons aux libraires vous laissant une marge bénéficiaire intéressante $»^{17}$.

On ne dispose pas de données permettant de mesurer l'importance du marché québécois pour l'éditeur parisien; tout au plus, peut-on s'en faire une idée par les commandes que la procure de Montréal passe à la maison-soeur de Paris. En 1962, une fois le contentieux des «livres de guerre » réglé, la procure de Montréal commande à Ligel 30000 livres de l'élève et 3000 livres du maître d'un manuel de géométrie de même que 10000 livres de l'élève d'un manuel de physique; Ligel devra lui accorder l'exclusivité des ventes de ces deux volumes au Canada et ajouter en couverture les mentions des deux librairies des frères au Canada: celles de Montréal et de Québec; Ligel accepte ces conditions mais seul le manuel de géométrie - Cours de géométrie portera la double mention, Paris disposant d'un stock suffisant du manuel de physique pour alimenter le marché québécois sans procéder à une réimpression. L'année suivante, les frères de Montréal commandent encore à leurs confrères de Paris 10000 exemplaires du manuel de géométrie et 1000 d'un manuel d'arithmétique ${ }^{18}$. En 1965, Lidec importe encore des manuels de géométrie et de physique - la lettre retracée fait état de cette commande sans préciser la quantité - et rachète de Beauchemin un stock de manuels de littérature - «Littérature vivante » de Louis Chaigne - édités par Ligel et dont Beauchemin assumait jusque-là la diffusion au Canada. Ces quelques données, si fragmentaires qu'elles soient, permettent d'entrevoir la place importante que les frères des Écoles chrétiennes de France ont occupée dans le marché du manuel scolaire québécois entre 1950 et 1970 et le rôle qu'ont joué leurs confrères québécois dans cette diffusion; elles expliquent aussi les nombreuses questions que la maison de Paris posera à celle de Montréal quand elle apprendra que leurs confrères outre-Atlantique 
ne les ont pas avertis des livres français qu'ils avaient réimprimés au Canada pendant la guerre, et même après ${ }^{19}$.

\section{Auteurs français rédigeant au Québec}

Une autre forme de l'influence de la France dans les manuels scolaires de la communauté au Québec doit être prise en compte: les auteurs français de manuels rédigés au Québec. Tant que la relève locale sera insuffisamment nombreuse, les premiers manuels rédigés au Québec le seront par des confrères immigrés de France : leur origine métropolitaine ne peut que transparaitre dans la rédaction des nouveaux livres ${ }^{20}$.

17 Le frère Adelbertus (Pierre-Louis Legendre) - un des quatre fondateurs de 1837 serait l'auteur de Série de questions sur les principales vérités de la religion (1869), de Manuel du catéchiste ou questionnaire avec réponses sur la doctrine chrétienne (1878 ?) et de Questionnaire explicatif du petit catéchisme de la province ecclésiastique de Québec (1881) ${ }^{21}$. Il semble avoir également rédigé Nouvelle géographie primaire illustrée à lusage [sic] des écoles chrétiennes de la puissance du Canada (1876) et Nouvelle géographie intermédiaire illustrée à l'usage des écoles chrétiennes de la puissance du Canada (toutes deux publiées initialement en 1876 et toutes deux traduites ultérieurement en anglais); encore en 1876, le frère Adelbertus aurait publié Géographie illustrée : cours moyen également traduit en anglais et une série de trois cahiers d'exercices cartographiques (c. 1882) ${ }^{22}$.

18 Autre cas. Arrivé au Québec par suite des décrets Combes ${ }^{23}$, le frère Romez, longtemps professeur au principal collège des frères dans la région de Montréal, le Mont-Saint-Louis, se voit attribué, particulièrement par les bibliothécaires de la Bibliothèque nationale du Québec, sinon la rédaction tout au moins la révision d'un certain nombre de manuels : Lectures courantes - deuxième livre (1916), Lectures graduées troisième livre (1917), Lectures littéraires et scientifiques - quatrième livre (1921), Arithmétique - cours moyen (troisième et quatrième année (1925) et Arithmétique - cours primaire supérieur (1925 également). En ce qui regarde les deux derniers titres, nous disposons de témoignages de l'auteur. L'exemplaire du Cours primaire supérieur conservé aux archives de la communauté à Rome porte une dédicace signée « de l'auteur Frère Romez »; l'année précédente il écrivait au directeur de la procure générale de Paris : "Je viens de terminer un cours moyen d'Arithmétique pour nos écoles du district de Montréal, et l'on me demande de me remettre immédiatement à l'ouvrage pour rédiger le cours élémentaire qu'on désire imprimer en juillet prochain. Pour m'aider dans ce travail, je vous serais très obligé si vous vouliez bien m'envoyer trois ou quatre exemplaires de cours élémentaire d'Arithmétique publiés par diverses librairies et

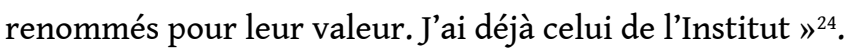

Présence donc du manuel scolaire de France au Québec par l'intermédiaire de cette communauté, présence qu'un certain nombre de facteurs expliquent, en premier lieu, la rareté. Jamais étudiée, celle-ci fait l'objet de trop de mentions dans la correspondance du Département de l'instruction publique au XIXe siècle pour qu'on puisse la reléguer au rayon des mythes ${ }^{25}$. Et même au XXe siècle, lorsque les frères de Montréal réimpriment, durant la Seconde Guerre mondiale, une quinzaine de manuels rédigés par leurs confrères de France, il s'agit vraisemblablement de combler un vide : la guerre a imposé un blocus aux importations européennes destinées aux collèges 
classiques et la production locale ne peut satisfaire aux besoins du secondaire public en pleine expansion ${ }^{26}$. Pourtant, c'est surtout dans la dialectique des deux forces en présence - l'État régulateur et la communauté producteur - qu'il faut chercher les principales explications du phénomène.

\section{L'exportation de manuels : les facteurs d'une politique}

$20 \mathrm{Au}$ moment où la communauté s'implante au Québec en 1837, aucune législation n'encadre la production ou la consommation du manuel scolaire, l'enseignant utilisant ce que bon lui semble: des manuels, certes, mais aussi des revues pieuses et des journaux. Il faut attendre 1860 et la mise en place du Conseil de l'instruction publique pour voir poindre une timide tentative de réglementation du manuel scolaire par la politique d'approbation. Bien sûr, cet organisme réclame des livres indigènes canadiens disait-on - mais pendant longtemps il s'agira de vœux pieux ; à preuve, sur ses 26 manuels d'origine française réimprimés au Québec durant le XIXe siècle, la communauté ne demande l'approbation que pour sept et ne subit aucune tracasserie administrative quant à la production et à la commercialisation des 19 autres $^{27}$. Ce laisser-faire quant au manuel étranger perdure jusqu'au milieu du XXe siècle, comme en témoignent l'impression à Montréal de la quinzaine des manuels français à partir de 1940 et le commerce que la maison de Paris de la même communauté entretient au vu et au su du gouvernement avec la douzaine de libraires québécois.

21 En face du pouvoir politique qui a voulu interdire - tel était, à défaut de législation contraignante, le discours officiel - ou tout au moins diminuer l'utilisation du manuel scolaire étranger en invoquant entre autres raisons l'uniformité, s'est retrouvé - et parfois dressé - un autre pouvoir qui a défendu ses propres politiques, en invoquant une autre uniformité, la sienne.

\section{Les raisons du soutien de la communauté}

Quel que soit l'état du marché des manuels scolaires ou de la réglementation qui en régit la distribution, la communauté qui arrive de France a un autre motif de s'en remettre à ses propres livres : l'utilisation de méthodes pédagogiques uniformes, et des livres qui en sont une des manifestations les plus tangibles, renforce chez ses membres le sentiment d'appartenance. De plus, comme l'attribution des tâches relève de la communauté, l'utilisation des mêmes livres facilite la mobilité des effectifs en réduisant au minimum la période d'adaptation des sujets ${ }^{28}$. Enfin, l'institut, en utilisant ses propres publications, est assuré de l'orthodoxie. La circulaire $n^{\circ} 107$ (1840) du supérieur général, le frère Philippe, est explicite au sujet de la politique de la communauté concernant les manuels : « [les frères] sont invités à n'en pas adopter d'autres, afin de conserver parmi nous l'uniformité si nécessaire au bon ordre et à la bonne direction des Écoles. ${ }^{29}$ On comprend alors que la congrégation vise à utiliser ses propres instruments pédagogiques, quel que soit l'endroit où elle œuvre.

Les responsables de la rédaction des manuels au Québec tiendront un discours similaire pendant un siècle. L'auteur d'un mémoire rédigé à Montréal en 1881 conclut: " les membres de cette commission [de rédaction des manuels] émettent le vœu que les Commissions-soeurs assemblées aux États-Unis, procèdent d'une manière analogue à la 
rédaction de leurs classiques, afin que dans toute la grande famille du Vénérable de la Salle il y ait, dans l'enseignement, unité de méthodes, de procédés, comme il y a unité de sentiments d'intention et d'aspiration. $»^{30}$ Soixante-dix ans plus tard, les nouveaux membres du même comité exprimeront une préoccupation analogue sous la plume du frère Raymond Brisebois: "Nos livres ont été rédigés conformément aux méthodes modernes les plus sûres mettant en valeur les procédés les plus récents. Ils ont néanmoins leurs racines dans le patrimoine très riche des travaux des anciens frères et de la tradition de l'Institut. $»^{31}$ Cette volonté de l'autorité française d'imposer ses propres manuels ne serait-elle pas une manifestation de néo-colonialisme? Devant le refus du Conseil de l'Instruction publique d'approuver les manuels des frères pour l'enseignement du dessin et de la grammaire, le supérieur au Québec, français d'origine, répondra en 1884 par un long plaidoyer articulé autour du succès que ces manuels ont connu dans leur version originale outre-Atlantique : ce qui est bon en France est bon au Québec ${ }^{32}$.

Il faut introduire une autre donnée pour expliquer le succès des manuels des frères de France au Québec, particulièrement durant le XIXe siècle. En dépit de la rupture des liens politiques avec l'ancienne métropole, la France avait continué d'influencer la pensée québécoise. Mais de quelle France s'agit-il ? Un long mémoire anonyme publié en 1886 - L'éducation ou la grande question sociale du jour: recueil de documents propres à éclairer les gens de bonne foi - montre combien les ultramontains québécois se méfiaient de l'influence de la France laïcisante : on y dénonce le surintendant de l'instruction publique, Gédéon Ouimet, et le directeur des écoles de la commission scolaire de Montréal, Urgel Archambault, qui ont patronné la visite de deux institutrices françaises déléguées par Jules Ferry et on y met en parallèle des textes officiels du ministère de l'Éducation de France et du département de l'Instruction publique du Québec pour montrer combien ce dernier, tout catholique qu'il soit, s'inspire d'un modèle laïque. Or, pour les ultramontains, la communauté d'origine française présente une garantie formelle d'orthodoxie; elle véhicule, particulièrement dans ses manuels, la pensée traditionnelle de l'Église catholique et, en conséquence, rien ne s'oppose à l'utilisation de la littérature didactique lasallienne. Ce n'est pas un hasard si l'auteur anonyme du mémoire rappelle, dans une charge, la "guerre de corsaire » que les inspecteurs d'école feraient au Traité des devoirs du chrétien. Et le défenseur du droit de la communauté à utiliser ses manuels, et tout particulièrement ceux publiés initialement en France, le bouillant frère Réticius - français d'origine mais supérieur de la communauté pour le Canada - est vu comme l'un des défenseurs des théories ultramontaines, à l'exemple de Mgr Laflèche, évêque de Trois-Rivières avec qui il fait cause commune.

Malgré la politique des autorités métropolitaines incitant les frères du Québec à utiliser les mêmes manuels que leurs confrères français, tout au moins dans les matières qui s'y prêtaient, malgré l'appui de la droite ultramontaine réclamant la non ingérence dans la pédagogie des communautés, nommément celle des frères des Écoles chrétiennes ${ }^{33}$, ces derniers subissent très tôt des pressions les incitant à rédiger des textes plus adaptés au milieu et répondant d'une façon plus adéquate aux impératifs des programmes scolaires québécois, ce qui suppose l'assentiment des autorités françaises de la communauté. Aussi les frères du Québec doivent-ils composer pour trouver une solution acceptable à tous.

Le frère Aphraates, fondateur de l'académie de Québec, ouvre le débat avec Paris en janvier 1881 ; « rapporteur de la commission des classiques », il a déjà assumé l'édition 
québécoise d'une demi-douzaine de manuels français et en a rédigé ou adapté une quinzaine d'autres. Il explique la situation particulière dans laquelle se trouvent les frères québécois. L'argumentation du religieux en faveur d'une adaptation des manuels de France repose sur deux postulats: 1.l'obligation de se conformer aux normes édictées par les organismes chargés d'approuver les manuels québécois; 2.la concurrence des auteurs laïques : comme ces derniers ne peuvent compter sur un fonds français, ils en arrivent, par la force des choses, à rédiger des manuels assortis de références au milieu, ce qui leur donne un avantage certain ${ }^{34}$.

En regard du premier argument, rien ne permet au frère Aphraates de prévoir que le discours officiel sur la restriction des manuels importés ne sera jamais renforcé par des sanctions effectives, malgré les nombreuses menaces de couper les fonds aux commissions scolaires utilisant des livres non approuvés ${ }^{35}$. C'est le deuxième postulat qu'il importe surtout de relever. Contrairement à ce que sous-entend le rapport, les auteurs laïcs n'ont pas attendu l'annonce des politiques de canadianisation pour se mettre à publier des manuels et rien ne permet d'affirmer qu'à partir des années 1880 , ils augmentent sensiblement leur part du marché au détriment des communautés religieuses, essentiellement les frères des Écoles chrétiennes à ce moment; en fait, si l'on s'en tient aux seuls manuels approuvés, cette décennie inaugure la mainmise des communautés religieuses sur la production et la distribution du manuel scolaire. L'utilisation du mot "laïque » dans un rapport destiné aux autorités parisiennes pouvait relever d'une stratégie : la lecture qu'on en ferait à Paris - Ferry commence à instaurer l'école laïque synonyme de neutre - différait totalement de celle du Québec où les professeurs dits " laïcs» ne peuvent s'afficher comme «neutres " ${ }^{36}$. Comme c'est Paris qui édicte les normes auxquelles doivent se plier les frères du Québec et qui accorde des dérogations, l'ambiguïté du terme ne pouvait qu'inciter les autorités parisiennes à accéder aux demandes de leurs confrères québécois : pour Paris, des livres rédigés par des auteurs laïcs ne présentaient pas des garanties absolues d'orthodoxie.

Jugeant que Paris ne répond pas assez vite, le frère Réticius revient à la charge en août, d'autant plus qu'il soupçonne le frère directeur de la procure parisienne de faire entrave au projet canadien. Aux arguments du mémoire de janvier, il ajoute des exemples concrets pour illustrer le bien-fondé de la demande canadienne : le système de mesure métrique rend inopérants les manuels de mathématique ${ }^{37}$, les géographies de France ne tiennent pas compte de la réalité nord-américaine, les grammaires sont trop élaborées compte tenu du temps imparti à l'enseignement de la langue française face à la nécessité d'enseigner aussi l'anglais, la grammaire des Clercs de Saint-Viateur rédigée au Québec risque de supplanter le manuel de sa communauté, et enfin les coûts de revient sont trop élevés dans le cas des manuels importés de France. Ne recevant toujours pas de réponse, Réticius reprend presque mot à mot son exposé quelques semaines plus tard, ajoutant cette fois une citation de la loi des droits d'auteur ! ${ }^{38}$ Deux réponses à l'automne 1881 donnent raison aux frères du Canada : permission leur est accordée de rédiger leurs manuels comme le font déjà leurs confrères d'Italie, d'Espagne et de Belgique ${ }^{39}$. La volonté des autorités françaises d'exporter leur littérature se fondait sur un certain nombre de postulats déjà énoncés, auxquels il faut ajouter la dimension proprement commerciale de l'opération.

29 L'apport français dans les manuels québécois comportait un avantage pécuniaire majeur : on évitait ou allégeait, dans les cas de réimpression ou d'adaptation, le coût de 
rédaction. Cette collaboration à l'intérieur de la communauté avait été prévue par les plus hautes instances administratives. Les frères de France confiaient initialement l'édition et la diffusion de leurs manuels à des maisons reconnues, notamment Mame de Tours et Poussielgue de Paris; ces éditeurs, ou libraires, se voyaient assurer, par contrat, le droit exclusif d'impression et de distribution des manuels de la communauté en France. Cependant, grâce à l'insertion d'une clause, les frères du Québec peuvent échapper au monopole consenti à Mame; notamment, on y avait inscrit le droit pour les frères hors de France d'éditer et de vendre les mêmes manuels à la seule condition de se tenir hors du marché métropolitain; Mame tente bien en 1903 de restreindre cette dérogation aux seuls ouvrages en langue autre que le français, mais l'administration de la communauté en France rejette cette prétention ${ }^{40}$. Dès lors, les frères du Québec croient pouvoir agir en toute liberté. Ces règles du jeu sont-elles si claires? Prise à la lettre, la restriction précitée permet aux frères hors de France d'imprimer des manuels, mais pour "l'usage de leurs écoles». Or, la clientèle de la procure de Montréal déborde rapidement le secteur des écoles dirigées par les Frères pour couvrir l'ensemble du marché scolaire. Mame et Poussielgue ignorent-ils cette entorse à la lettre du contrat? On ne trouve aucune trace d'une quelconque récrimination de leur part ${ }^{41}$. En plus de leurs propres productions, les Frères du Québec peuvent donc imprimer comme bon leur semble les manuels rédigés par leurs confrères de France sans la moindre contestation des éditeurs français; celle-ci viendra, mais d'une direction que n'avait pas prévue la procure de Montréal.

\section{Obstacles et réserves}

Depuis le début du XXe siècle, les frères de France ont transformé radicalement leur stratégie en regard des manuels scolaires qu'ils rédigent, et les décrets Combes avec leur corollaire inévitable - chute dramatique de leur clientèle - ne sont pas étrangères à ce chambardement. Ils commencent d'abord par créer une firme prête-nom, la Société générale d'édition, qui prend en charge l'édition de leurs manuels scolaires. Les frères n'ont maintenant plus d'intermédiaires entre la production et la vente; l'élimination d'un éditeur étranger permet à la communauté française d'escompter une meilleure marge de bénéfice, mais entre temps, elle les oblige à assumer seuls les coûts d'édition à un moment où le marché français leur est moins accessible alors qu'augmentent les coûts de production dans l'immédiat après-guerre.

Pour rétablir sa situation, la procure de Paris en arrive à proposer aux autorités de l'institut, dans un mémoire en date du 20 avril 1925, l'établissement de droits compensatoires pour les manuels de France copiés ou adaptés par leurs confrères dans d'autres pays; on fonde l'argumentation sur la notion du droit d'auteur: la procure de Paris ayant investi dans la rédaction des manuels - on parlera bientôt de "frais de premier établissement »-il est normal que les procures hors de France, auxquelles ces dépenses ont été épargnées, contribuent à éponger ces frais par un système de redevance comme elles le feraient si les manuels avaient été publiés par des éditeurs laïcs. La plus haute instance administrative de l'institut entérine l'essentiel des recommandations du rapport de la procure de Paris : $10 \%$ du prix de vente pour les réimpressions et $5 \%$ pour les adaptations ${ }^{42}$.

Il semble bien que le principe des redevances ne sera pas contesté : il s'agit d'une mesure administrative interne à une communauté religieuse qui, tout en se référant à 
des conventions légales, relève aussi de principes étrangers aux seules opérations commerciales; un réquisitoire de deux pages en date du 12 juin 1927 et émanant vraisemblablement de la procure de Montréal conteste jusqu'à la valeur « juridique » de la notion de droit d'auteur utilisée par la procure de Paris ${ }^{43}$, mais dans la pratique, on se soumettra au principe de redevance. Les vrais problèmes surgissent lorsqu'il faut déterminer à partir de quelle date s'applique la nouvelle directive et quels livres sont sujets à redevances : si les reproductions intégrales ne portent pas à discussion, il en va autrement pour les adaptations. Et comme la similitude des titres peut prêter à confusion, on crée une "commission chargée de comparer les livres classiques de l'Institut ». Le moins qu'on puisse dire, c'est que cette commission - qui siège à Paris et dont tous les membres sont vraisemblablement français - remplit sa tâche avec zèle ${ }^{44}$. Finalement, après deux ans de discussion et d'échanges épistolaires parfois acrimonieux, les cas litigieux sont tranchés par la plus haute instance de l'Institut.

Même après révision ultérieure de la précédente liste, le catalogue d'édition de la procure de Montréal pour la période 1925-1940, ne compte plus qu'une dizaine de titres qui, de l'aveu des deux parties, se rattachent au fonds français initial; et encore certains, comme une grammaire du XIXe siècle, ne touchent plus qu'un marché infime. Il n'en demeure pas moins qu'un nouveau principe a été accepté : contrairement au XIXe siècle, les procures locales ne peuvent plus utiliser librement le fonds français et les nouvelles règles, sanctionnées par les plus hautes instances de la communauté, seront régulièrement prorogées. Quand les frères de Montréal « oublient » de verser les arrérages pour les manuels imprimés à partir de 1941, leurs confrères de Paris, appuyés par les supérieurs maintenant établis à Rome, les rappellent à l'ordre.

$34 \mathrm{Au}$ centre de l'enseignement public québécois pendant plus de cent ans, principal éditeur de matériel pédagogique pendant plus de cinquante ans, la communauté des Frères des écoles chrétiennes s'est également imposée comme un des principaux agents de la pénétration du manuel scolaire de France. Ce succès de commerce international s'explique tant par la carence d'instruments pédagogiques au moment où émigre la communauté que par des facteurs d'ordre structurel - elle préfère véhiculer ses propres textes pour fin d'uniformisation et d'orthodoxie - sans oublier les retombées économiques suffisamment importantes pour engendrer occasionnellement des frictions à l'intérieur de ses propres rangs.

Une fois planté le décor dans lequel s'est joué ce transfert, il faudra analyser l'impact des contenus. Ainsi, qu'en est-il du message religieux de ces manuels importés de France : a-t-il eu une quelconque influence sur les mentalités et les comportements d'une société où la majorité francophone adhérait officiellement au catholicisme? Un seul exemple suffit à illustrer l'importance de la question. Le plus grand succès de librairie des frères - plus de soixante réimpressions au Québec entre 1841 et 1904 venait de France et se voulait une somme de théologie populaire : Le nouveau traité des devoirs $d u$ chrétien. Officiellement catalogué comme livre de lecture, son contenu apologétique était tel que les protestants craignaient qu'on en imposât la lecture à leurs candidats au brevet d'enseignement ${ }^{45}$. Il était systématiquement accompagné d'un autre livre relié sous la même couverture et également d'origine française, Les règles de la bienséance et de la civilité chrétienne; dans ce dernier cas, non seulement on tente 
d'utiliser un manuel pour la propagation d'une façon de penser, chrétienne, mais aussi d'une façon de se comporter, métropolitaine.

Les effets qu'a pu avoir cette importation massive sur les habitudes de lecture au Québec restent à analyser. Reportons-nous une fois de plus au même Nouveau traité des devoirs du chrétien. Selon le surintendant Chauveau, il "parait être lu dans 193 municipalités » en $1855^{46}$; l'inspecteur Lanctot écrit trois ans plus tard: «[...] après deux ou trois ans d'école, il n'y a pas un éleve [sic] qui ne l'ait lu et relu dix fois, il perd donc ainsi de son intérêt et l'élève cesse d'éprouver de l'attrait pour la lecture. Il lit non plus dans l'espoir de satisfaire sa curiosité naturelle, d'apprendre quelque chose de nouveau, mais uniquement par routine et pour s'acquitter de la tâche qu'on lui impose $\aleph^{47}$. En réponse aux jérémiades du supérieur quant aux tentatives qu'on ferait d'en interdire l'usage, le surintendant sent le besoin de préciser : "Je pourrais peutêtre dire ici que certaines personnes se sont trompées en croyant que je recommandais ces livres [les livres de lecture de l'auteur québécois Montpetit] à l'exclusion du Devoir $d u$ Chrétien, depuis longtemps populaire. Je n'exclus pas ce dernier ouvrage; je dis seulement qu'il est de peu de valeur pour l'enseignement de la lecture. Il n'est pas gradué, et il est d'un genre trop élevé pour l'enfance [...]. » ${ }^{48}$

Les interrogations soulevées autour des pratiques éditoriales des frères des Écoles chrétiennes gagneraient à être reprises pour chacune des communautés religieuses françaises qui se sont implantées au Québec. Signalons, à titre d'exemple, le cas des Frères de l'instruction chrétienne dits frères de Ploërmel : L'agriculture dans les écoles : 41 leçons qu'ils publient à Montréal en 1896 est la réimpression presque littérale d'un texte publié initialement en Bretagne. Au fil des rééditions qui s'échelonnent jusqu'à 1940, l'ouvrage a-t-il été adapté aux exigences de l'économie, du climat et du sol québécois ? Voilà une question parmi tant d'autres qui mériteraient d'être étudiées afin de cerner l'ajustement de l'offre française à la demande québécoise.

\section{NOTES}

1. Cette étude, dont une première version a été présentée au congrès de l'International Standing Conference for the History of Education à Maynooth (Irlande) en septembre 1997, s'inscrit dans le cadre du projet «École et société au Québec: histoire des contenus scolaires » subventionné par le Conseil des recherches en sciences humaines et auquel collaborent également Thérèse Hamel (Université Laval) et Serge Gagnon (Université du Québec à Trois-Rivières). Je remercie ce dernier qui a bien voulu revoir ce texte. Abréviations: AFEC pour Archives des Frères des écoles chrétiennes, Montréal, Paris ou Rome suivant le cas.

2. Pour l'histoire de la communauté au Québec, on se reportera à Nive Voisine: Les Frères des Écoles chrétiennes au Canada, t. 1 : La conquête de l'Amérique 1837-1880, Québec, Anne Sigier, 1987, 443 p. ; t. 2 : Une ère de prospérité 1880-1946, Québec, Anne Sigier, 1991, 471 p. ; t. 3 : Inquiétudes et renouvellement 1946-1987, Québec, Anne Sigier, 1999, 407 p.

3. Le catalogue des manuels scolaires québécois est en voie de compilation. Voir Paul Aubin: Manuels scolaires québécois. [En ligne : http://www.bibl.ulaval.ca/ress/manscol/]. Pour un état des connaissances sur l'histoire du manuel scolaire québécois, on se reportera à Paul Aubin : Le 
manuel scolaire dans l'historiographie québécoise, Sherbrooke, Université de Sherbrooke, Groupe de recherche sur l'édition littéraire au Québec, 1997, 151 p.

4. Frère Aidant au très vénérable frère, 22 mars 1838, AFECR, boîte 432a, no 141. Rien ne prouve que la requête a été exaucée.

5. Classiques canadiens - Autorisation demandée d'imprimer nos classiques en Canada, AFECR, boîte 411, dossier no 8, 2 .

6. Signalons un cas d'espèce dans le processus de diffusion des manuels scolaires français de cette communauté au Québec. On trouve, dans la collection des livres rares de l'université Laval, Exercices orthographiques d'analyse grammaticale, d'analyse logique [...] de «F.P.B.» (supérieur des Frères des écoles chrétiennes à Paris) et publié à Montréal en 1858 par L.J. Prégen; or cette identification de l'éditeur montréalais est imprimée sur un papillon collé au bas de la page de titre sous lequel on peut lire "Tours, Mame »; il semble que l'éditeur Prégen a acheté de Mame (principal éditeur de la communauté en France) un certain lot de cette grammaire et qu'il a voulu l'écouler sur le marché québécois en faisant croire qu'il s'agissait d'une publication locale ; rien ne nous permet de penser que la communauté a été impliquée dans cette opération.

7. Abrégé de l'exposition de la doctrine chrétienne;Leçons de langue française;Précis d'histoire littéraire;Cours abrégé de littérature;Cours d'algèbre conforme aux derniers programmes de l'enseignement secondaire - classes de seconde, de première et de philosophie;Exercices d'algèbre;Premières notions de sciences à l'usage des élèves se préparant au certificat d'études primaires;Notions sur les sciences physiques et naturelles;Cours d'histoire naturelle;L'Antiquité et le Moyen-Âge;Les temps modernes et l'époque contemporaine;Simples notes explicatives des tableaux silhouettiques sur la vie de Jésus; Histoire sainte et vie de N.-S. Jésus-Christ ;Histoire de l'Église depuis sa fondation. La plupart des «manuels de guerre» utilisent, pour désigner l'auteur, l'expression «Une réunion de professeurs », formule par laquelle les frères des Écoles chrétiennes de France signaient leurs livres depuis déjà plusieurs décennies.

8. Dans sa description de la quatrième édition, Bibliographie du Québec (7-602) apporte la précision suivante : «Éd. originale, Paris : Librairie Vic et Amat, 1914, publiée sous le titre : Dictionnaire de bon langage ». Nous sommes en présence d'un des rares cas où la communauté québécoise publie un manuel dont l'auteur n'est pas un de ses membres.

9. Fr. Oswald, proc. Liste d'ouvrages de l'Institut ayant fait l'objet d'une adaptation et d'une reproduction 20 mars 1926, AFECP, Montréal, droits d'auteur 1925. Un manuel français au titre identique avait déjà été réimprimé au Québec entre 1873 et 1911 mais ne semble pas être la source de celui de 1925.

10. «Après avoir examiné l'arithmétique en usage dans les Écoles publiques, je n'en ai trouvé aucune qui soit comparable à la nôtre pour la clarté des méthodes ; je me suis décidé à en extraire la théorie en changeant les mètres en aunes ou en toises selon l'occurrence, les francs en Louis dont on fait usage ici. » F. Aidant au très vénérable frère, 22 mars 1838, AFECR, boîte 432a, $n^{\circ} 141$. 11. On peut supposer que les frères de Montréal avaient en main, dès 1872, un exemplaire de ce syllabaire français succinct dont ils se sont inspirés pour publier leur manuel; en 1875, ils auraient décidé de publier in extenso la version française plus courte et qui pouvait passer pour un abrégé de la production de 1872.

12. Poème publié d'abord à Paris en 1887 et ensuite à Québec en 1890 (voir David Hayne : «La légende d'un peuple », Dictionnaire des ceuvres littéraires du Québec, Tome premier des origines à 1900, Montréal, Fides, 1978, p. 445). Vraisemblablement la première citation de cette œuvre de Fréchette dans un manuel scolaire.

13. Frère Manuel-Paulin: Liste des Ouvrages de l'Institut ayant fait l'objet d'une Adaptation et d'une Reproduction Année 1935, AFECM, T58 C39.

14. [Frère Réticius], Aux honorables membres du comité catholique du conseil de l'instruction publique, s.l., s.n., [1884], p. 1. Paul de Cazes au frère Réticius, 3 octobre 1885, Archives nationales du Québec, Éducation - Correspondance expédiée, M93/44. 
15. Paul Aubin et Michel Simard: Les manuels scolaires dans la correspondance du Département de l'instruction publique 1842-1899, inventaire, Sherbrooke, Université de Sherbrooke, Groupe de recherche sur l'édition littéraire au Québec, 1997, entrée n 950.

16. On peut consulter aux AFECM une collection de manuels imprimés en France. Leur présence au Québec ne signifie pas automatiquement leur importation en vue de les écouler sur place : il peut s'agir, dans bien des cas, d'exemplaires emportés dans les bagages de tel ou tel individu. Dans le cas qui nous occupe, il semble bien qu'il y ait eu diffusion commerciale de l'un ou l'autre des livres de dessin dont on trouve des exemplaires dans ces mêmes archives. En plus des cinq inspecteurs identifiant formellement l'utilisation de manuels de dessin rédigés par la communauté qui nous occupe, deux autres, dont celui de l'Assomption, soulignent que chaque communauté utilise sa propre méthode de dessin; cette pratique de permettre à chaque communauté religieuse d'employer ses propres manuels - approuvés ou pas - ouvrait aux frères des Écoles chrétiennes un marché intéressant.

17. AFECP, Dossier LIGEL Édition Litige avec Montréal. Visiblement, le frère directeur de Ligel ignore que ses confrères de Montréal réimpriment des manuels français depuis 1941, et rien n'indique que le directeur de la procure de Montréal l'ait renseigné par la suite ; cet « oubli » lui sera remis en mémoire dans quatre ans. Dans une autre lettre également datée de 1958, le directeur de la maison parisienne affirme compter parmi sa clientèle une dizaine de distributeurs québécois, dont Fides, Granger, Beauchemin et Dussault.

18. On ne sait pas quel était le titre exact de ce manuel d'arithmétique ; par ailleurs, sa diffusion au Québec semble avoir souffert de graves lacunes si l'on se reporte à une mercuriale adressée au directeur de Ligel par le frère Hector Gravel, professeur de mathématiques au Mont-Saint-Louis. AFECP, Dossier LIGEL Édition Litige avec Montréal.

19. Les informations proviennent des AFECP : dossier Procure 1900-1950 LIGEL (1924-1964) et dossier LIGEL Édition Litige avec Montréal.

20. Clara Brafman («Les manuels scolaires de lecture d'origine française en Argentine », Histoire de l'éducation, janv. 1996, pp. 63-80) prend en considération les livres rédigés en espagnol par des instituteurs français résidant en Argentine.

21. Nive Voisine : «Les Frères des Écoles chrétiennes et l'éducation de la foi », Sessions d'étude, 56, s.l., Société canadienne d'histoire de l'église catholique, 1989, pp. 74-75. Raymond Brodeur (Les catéchismes au Québec, 1702-1963, Sainte-Foy, Presses de l'université Laval, 1990, p. 186), sur la foi d'une note manuscrite sur la page de garde de l'exemplaire du Questionnaire explicatif conservé aux AFECM, reprend à son compte la même affirmation. Il faut bien avouer que l'habitude de l'anonymat dans les publications pédagogiques des communautés oblige à beaucoup de prudence quant à l'attribution des paternités.

22. Dans le cas présent, je me base sur l'enregistrement du copyright au nom du frère. Ici encore, et tout comme pour l'anonymat, il faut user de beaucoup de prudence. Tant que la communauté n'a pas de statut légal au Québec, elle fait enregistrer les droits d'auteur au nom d'un de ses membres afin de se prémunir de possibles plagiats, comme l'expérience le lui a apprise dès les années 1840; habituellement, on utilise le nom du supérieur local qui n'est jamais, ou presque, l'auteur alors qu'en France on portait systématiquement au crédit du supérieur général - comme le frère Philippe Bransiet : F.P.B. - la rédaction des manuels. Dans les quelques cas, au Québec, où le copyright est enregistré au nom d'un individu autre qu'un supérieur, il semble qu'on doive y lire une reconnaissance de son statut d'auteur. Français tout comme le frère Adelbertus mais arrivé quelques années plus tard, le frère Aphraates (J.-F.-N. Dubois) aurait lui aussi rédigé un bon nombre de manuels scolaires, toujours si l'on en croit à la fois la formulation du copyright et la tradition de la communauté.

23. Sur l'arrivée d'un nouveau contingent de frères des Écoles chrétiennes au début du siècle, voir Guy Laperrière : Les congrégations religieuses de la France au Québec, 1880-1914, Tome 2 : Au plus 
fort de la tourmente 1901-1904, Sainte-Foy, Presses de l'université Laval, 1999, pp. 450-460. « [...] on reconnaîtra volontiers [leur] grande culture et [leur] compétence intellectuelle » (p. 457).

24. Frère Romez au frère directeur, 24 décembre 1924, AFECP, Canada correspondance 1924-1943. Notons que l'auteur ne limite pas ses sources d'inspiration aux seuls manuels publiés par sa communauté.

25. Paul Aubin et Michel Simard: Les manuels scolaires dans la correspondance du Département de l'instruction publique 1842-1899, inventaire, 1997, 342 p. (Le « département » remplit la fonction d'un « ministère »).

26. Paul-André Turcotte: L'enseignement secondaire public des frères éducateurs (1920-1970), Montréal, Bellarmin, 1988, 220 p.

27. Pour le rôle des autorités quant à l'usage des manuels, voir Paul Aubin : L'État québécois et les manuels scolaires au XIXe siècle, Sherbrooke, Université de Sherbrooke, Groupe de recherche sur l'édition littéraire au Québec, 1995, 119 p.

28. Jusqu'en 1964, les communautés religieuses oeuvrant dans le secteur public de l'enseignement signent avec les instances locales - les commissions scolaires - des contrats collectifs leur laissant toute latitude pour désigner les individus devant combler les postes prévus dans l'entente.

29. Sous l'entrée «Arithmétique " dans Noms de quelques frères ayant collaboréà la composition des classiques de notre institut, AFECR, boîte KA 753, dossier $n^{\circ} 1$. Il existait bien, depuis le début du XIXe siècle, une réglementation sur l'usage des manuels scolaires en France, mais le laxisme dans son application permettait, dans les faits, à la communauté d'utiliser ses propres manuels ; voir Alain Choppin : Les Manuels scolaires: histoire et actualité, 1992, pp. 24-35.

30. Propositions et questions contenues dans le Rapport de la Commission [...], AFECM, T58 C66c.

31. Comité des livres 1946-1955, AFECM, T58 C40.

32. [Frère Réticius], Aux honorables membres du comité catholique du conseil de l'instruction publique, s.l., s.n., [1884], pp. 1-15.

33. "Plusieurs de ces congrégations ont leurs livres, leurs manuels, leurs méthodes. Ces livres, ces méthodes sont des éléments constitutifs de la formation pédagogique qu'elles donnent à leurs membres, dans leurs noviciats ou dans leurs juvénats. Et, après leur temps d'épreuve, ces instituteurs et institutrices congréganistes enseignent naturellement avec les livres et d'après les méthodes de leurs instituts. Il est manifeste que l'État n'a aucun droit sur les constitutions, les règlements, la direction pédagogique de ces congrégations »: Thomas Chapais : Discours sur la loi de l'instruction publique prononcé par l'honorable M. Chapais devant le Conseil législatif, les 2 et 3 mars 1899, 1899, p. 10.

34. Propositions et questions contenues dans le Rapport de la Commission chargée d'examiner l'opportunité du remaniement des classiques - (Commission tenue au Canada, en janvier 1881), AFECM, T58 C66c.

35. Une commission scolaire locale compte sur trois sources de revenus : la taxe foncière payée par les propriétaires, la rétribution mensuelle payée par les parents au prorata du nombre d'enfants fréquentant les écoles et la subvention du département de l'Instruction publique; on ne connaît aucun cas - malgré de nombreuses menaces - où une commission scolaire aurait été privée de sa subvention gouvernementale pour utilisation de manuels non approuvés.

36. Mémoire présenté par les instituteurs laïques catholiques de la province de Québec à leurs seigneurs les Évêques de la dite province, faisant partie du Conseil de l'instruction publique, s.l., s.n., [1877?], 4 p.

37. Dès son implantation en sol québécois la communauté avait effectué cette conversion: le premier manuel qu'elle fait publier moins de six mois après l'arrivée des premiers frères à Montréal, Nouveau traité d'arithmétique contenant toutes les opérations ordinaires du calcul, les fractions et les différentes réductions de fractions [...] (Montréal, C.P. Leprohon, 1838), était une reprise d'un texte publié initialement en France et où on avait effectué la conversion du système métrique aux mesures à l'anglaise. 
38. Classiques au Canada - Exposé des raisons qui militent en faveur de l'autorisation d'imprimer les Classiques au Canada, 12 octobre 1881, ARECR, boîte $n^{\circ} 411$, dossier n ${ }^{\circ} 8,5$.

39. Frère Armin-Victor au frère provincial, Paris, le 28 octobre 1881, AFECM, T28 C3; frère Irlide au frère assistant, Besançon, le 26 octobre 1881, AFECR, boîte 411, dossier $n^{\circ} 8,4$.

40. Traité avec MM. Mame \& fils, \& MM. Poussielgue frères. 19 mars 1869 à 1880 inclusivement, AFECR, boîte EB $162 \mathrm{n}^{\circ} 3$, dossier $\mathrm{n}^{\circ} 9$. Le traité de 1859 contenait une clause analogue. Correspondance entre librairies, observations diverses, etc., 1895-1908, AFECR, boîte EB 162, $\mathrm{n}^{\circ} 1$.

41. Mame compte le marché québécois dans sa clientèle, tout au moins pour ses éditions dont les frères de France ne sont pas les auteurs ainsi qu'en témoigne la correspondance qu'il échange durant le XIXe siècle avec le surintendant de l'instruction publique (voir Paul Aubin et Michel Simard: Les manuels scolaires dans la correspondance du département de l'Instruction publique 1842-1899, inventaire, « Mame, Alfred » à l'index) ; on peut donc difficilement croire qu'il aurait ignoré les réimpressions québécoises par la procure de Montréal de manuels qui étaient couverts par le contrat entre sa maison et les frères de France. La procure de Paris, sans aucun doute à tort, en arrive même à soupçonner Mame de vendre au Québec des manuels des frères de France, et ce jusque dans les écoles des frères. Frère directeur de la procure de Paris au frère Oswald, procureur de Montréal, 10 mai 1927, AFECM, T58 C37.

42. Droits d'auteur correspondance 1925-1927, AFECM, T58 C37.

43. Reproduction et adaptation de livres de la Procure Générale - Respectueuses considérations [...], AFECM, T58 C37.

44. « Notes sur quelques livres de français édités par la Procure de Montréal », AFECP, Montréal droits d'auteur - 1925.

45. John Hamilton Graham : Letters to the superintendant of éducation for Lower Canada, Montréal, John Lovell, 1865, p. 3.

46. P.-J.-O. Chauveau : Rapport du surintendant de l'éducation pour le Bas-Canada pour l'année 1855, Toronto, John Lovell, 1856, p. 15.

47. P.-J.-O. Chauveau : Rapport du surintendant de l'éducation dans le Bas-Canada, pour l'année 1858, Toronto, John Lovell, 1859, p. 174.

48. Gédéon Ouimet: Rapport du surintendant de l'instruction publique de la province de Québec pour l'année 1881-82, Québec, Charles-François Langlois, 1883, pp. XIII-XIV.

INDEX

Index géographique : Canada, France, Québec (province)

Index chronologique : XIXe siècle, XXe siècle

Mots-clés : congrégation enseignante, enseignement primaire, manuel scolaire, matériel didactique, transfert culturel

\section{AUTEUR}

PAUL AUBIN

Centre interuniversitaire d'études québecoises, Université Laval 